\title{
The role and efficacy of multidisciplinary council in the bone and soft tissue tumor patients
}

\author{
Hüseyin Sina Coşkun $\odot$, Nevzat Dabak®, Hasan Göçer $\odot$, Ferhat Say® \\ Department of Orthopedics and Traumatology, Ondokuz Mayls University School of Medicine, Samsun, Turkey
}

\begin{abstract}
Objectives: Bone and soft tissue tumors have become more common and recognized diseases with developing medical technologies. The life span of the patients has been prolonged with more effective treatment methods and developing technology. Regardless of their area of expertise, the concept of multidisciplinary tumor approach has emerged in recent years, since it is difficult for a single physician to manage a malignant mass treatment process. For this purpose, we aimed to share our multidisciplinary bone and soft tissue tumor council data results.

Methods: Patients who were evaluated at the Department of Orthopedics andTraumatology of Ondokuz Mayis University between January 2004 and June 2017 were evaluated retrospectively. For this study, the weekly archived tumor council forms were evaluated and the data were transferred to the computer via Microsoft Excel and SPSS programs. The database for the specified years was created and the results were evaluated and the database was evaluated.

Results: A total of 2788 patients were included in the study. After the patients with data deficiency were removed, 2397 patients were the subject of the study. In the evaluation of the first 1960 patients, 658 primary bone tumors, 577 primary soft tissue tumors, 356 cases of metastases and 374 non-tumoral cases were detected. The most common benign bone tumor was enchondroma, while the most common malignant bone tumor was osteosarcoma. The most common benign soft tissue tumor was lipoma, whereas the most common malignant soft tissue tumor was malignant mesenchymal tumor. The most common non-tumor cause was chronic infection. The diagnosis of 203 patients was different from the definitive diagnosis.

Conclusions: In this study, we determined the epidemiological distribution of the cases evaluated in the multidisciplinary tumor council in the Middle Black Sea and the role of multidisciplinaryapproach in treatment and survival has a positive effect especially in selected patient groups.

Keywords: Multidisciplinaryapproach, benign bone tumor, soft tissue tumor, sarcomas
\end{abstract}

$\mathrm{B}$ efore the modern treatment approaches in current use, amputation was the primary treatment method for the cases with a malignant bone or soft tissue tumor. With developments over time, adjuvant chemotherapy or radiotherapy was introduced to the treatment protocols of sarcoma patients. Even with these innovations on bone and soft tissue tumors field, the 5-year survival rates of these protocols were meager. In recent years, there has been an increase in the determination of musculoskeletal system tumors with

How to cite this article: Çoşkun HS, Dabak N, Göçer H, Say F. The role and efficacy of multidisciplinary council in the bone and soft tissue tumor patients. Eur Res J 2021;7(3):278-283. DOI: 10.18621/eurj.716555

Address for correspondence: Hüseyin Sina Çoşkun, MD., Assistant Professor, Ondokuz Mayls University School of Medicine, Department of Orthopedics and Traumatology, Samsun, Turkey.E-mail: sina.coskun@hotmail.com 
the development and widespread use of the diagnostic methods of computed tomography (CT) and magnetic resonance imaging (MRI) and these tumors are well recognized nowadays.

Experienced centers are at the forefront of diagnosis and treatment of musculoskeletal system tumors. When examined from the perspective of a single specialty, the diagnosis and treatment of malignant bone and soft tissue tumors is usually insufficient. In this context, the treatment of musculoskeletal system tumors started to be planned by a multidisciplinary council worldwide. With the multidisciplinary tumor council, delayed diagnosis of malignant sarcomas have been avoided, the time to initiation of treatment has been shortened, and there has been a positive effect on survival rates. The Council is formed of specialists from orthopedics and traumatology, pathology, radiology, nuclear medicine, radiation oncology, internal medicine, and pediatric oncology. In Turkey, a similar council method of working was first implemented by Güven Yücetürk et al. [1].

This study aimed to emphasize the importance of a multidisciplinary approach in the diagnosis and treatment of bone and soft tissue tumors by retrospectively evaluating the data of the database of Bone and Soft Tissue Tumour Council (BSTTC) of Ondokuz Mayis University School of Medicine between June 2004 and June 2017. It was also aimed to share the data of a bone and soft tissue tumor center.

\section{METHODS}

The study included patients evaluated by the BSTTC, which operates within the Orthopaedics and Traumatology Department of Ondokuz Mayis University School of Medicine. The study parameters were formed based on the BSTTC form, including patient name, age, gender, complaints, in brief, initial diagnosis, definitive diagnosis, and BSTTC decision. The study database was created from a retrospective scan of these data.

\section{Ethical Committee Approval}

Approval for this study was granted by the Clinical Ethics Committee of Ondokuz Mayis University (decision no: OMUKAEK 2017/259, dated: 20.07.2017). No financial support was obtained from any source for this study.

\section{Statistical Analysis}

Data obtained in the study were analyzed statistically using SPSS for Windows 21.0 software (SPSS Inc, Chicago, IL, USA). Descriptive statistics were presented as mean \pm standard deviation, median (minimum-maximum) values, frequency (n) and percentage $(\%)$

\section{RESULTS}

Between 2004 and 2017, a total of 2788 patients were evaluated by BSTTC of Ondokuz Mayis University School of Medicine. When the BSTTC evaluation forms were screened, the data of 391 patients were incomplete, so these patients were excluded from the study. The data of the remaining 2397 patients were, and it was determined that 437 patients were discussed more than once by BSTTC. These 437 patients were excluded, and the study sample was formed of 1960 patients who received an initial diagnosis of bone or soft tissue tumor. These patients comprised 996 males and 964 females with a mean age of 40.54 years (min 17 days-max 96 years). The primary bone tumor was determined in 658 patients, primary soft tissue tumor in 577 , metastatic disease in 356 , and non-tumoral conditions in 369 . The study parameters are shown in Table 1.

In some cases, a single patient was evaluated several times by BSTTC. For example, one patient with a diagnosis of osteosarcoma was discussed by BSTTC eight times. This situation was generally observed in patients diagnosed with malignant bone or soft tissue tumor. Sometimes evaluation of complications that developed in the treatment process and if necessary, a review of the treatment, meant that the patient was re-evaluated by BSTTC.

Taking this information into consideration, BSTTC re-evaluated $72(20 \%)$ of all the metastatic cases, 64 (53.33\%) patients with Ewing sarcoma, 48 $(66.66 \%)$ with osteosarcoma, $19(27.76 \%)$ with malignant mesenchymal tumour, $12(21.81 \%)$ with giant cell bone tumour, all the cases with atypical lipomatous mass (19 times, 190\%), 11 (44\%) patients with chondrosarcoma, $10(62.5 \%)$ with desmoid tumour, and $10(18.51 \%)$ with osteoid osteoma. 
Table 1. Details of the patients included the study

\begin{tabular}{|c|c|}
\hline & $\begin{array}{l}\text { The number of patients } \\
\text { (n) }\end{array}$ \\
\hline Patients evaluated by BSTTC 2004 and 2017 & 2788 \\
\hline Total number of patients after exclusion of those with incomplete data & 2397 \\
\hline Patients discussed more than once by BSTTC & 437 \\
\hline Total number of patients included the study & 1960 \\
\hline \multicolumn{2}{|l|}{ Gender } \\
\hline Male & 996 \\
\hline Female & 964 \\
\hline Mean age (years) & 40.54 \\
\hline Primary bone tumour & 658 \\
\hline \multicolumn{2}{|l|}{ Benign $(n=447)$} \\
\hline \multicolumn{2}{|l|}{ Malignant $(\mathrm{n}=211)$} \\
\hline Benign $(\mathrm{n}=353)$ & 572 \\
\hline \multicolumn{2}{|l|}{ Malignant $(\mathrm{n}=219)$} \\
\hline Metastatic disease & 356 \\
\hline Non-tumoral conditions & 374 \\
\hline
\end{tabular}

BSTTC $=$ Bone and Soft Tissue Tumour Council

Total number of patients

$\begin{array}{lllll}0 & 125 & 250 & 375 & 500\end{array}$

Benign Bone Tumors

Benign Soft Tissue Tumors

Malignant Bone Tumors

Malignant Soft Tissue Tumors

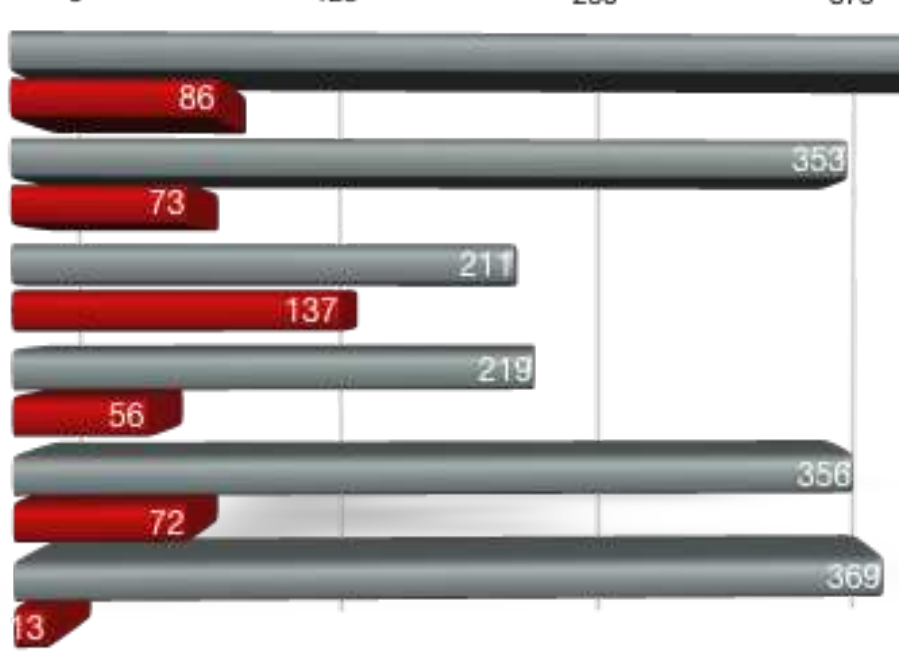

Fig. 1. The ratios of re-evaluated patients to the totals of all diagnosed patients. 


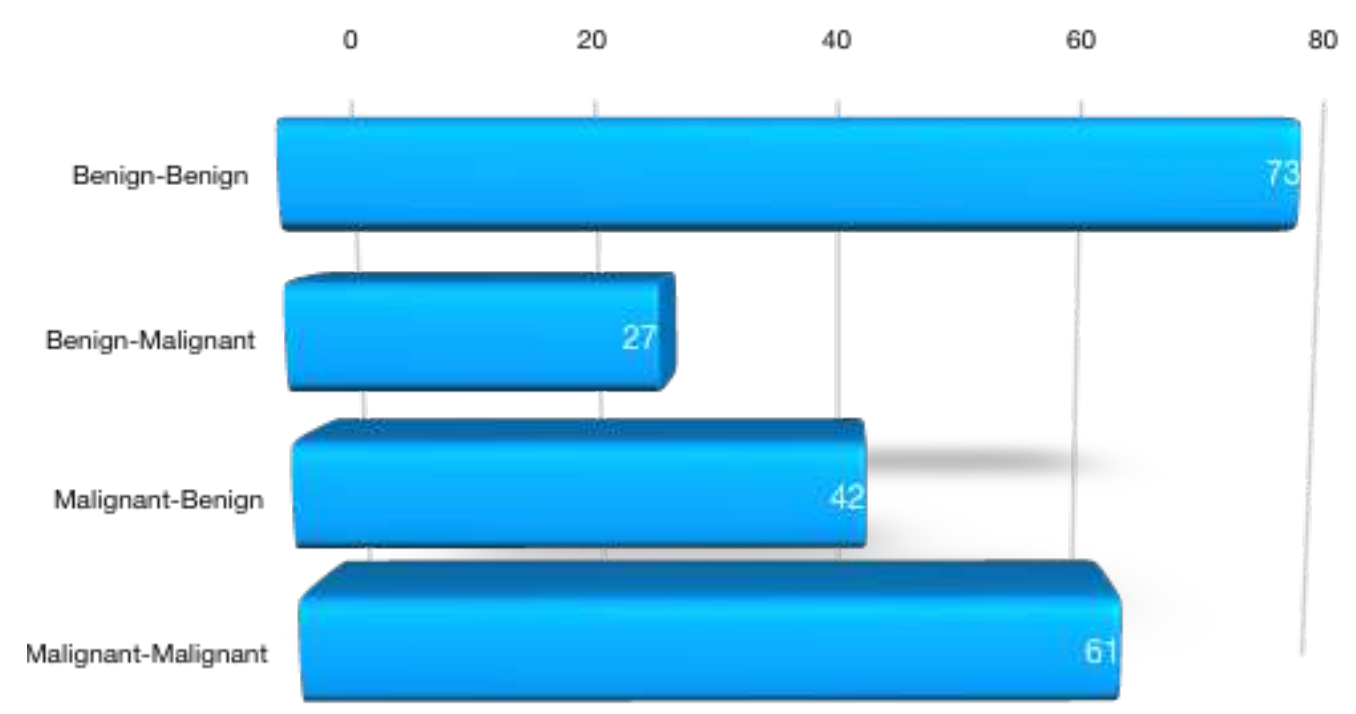

Fig. 2. Patients with a re-evaluation of diagnosis. The first word refers to the initial diagnosis of the patients and the second word to the definitive diagnosis.

In other words, re-evaluation was made of $86 / 447$ (19\%) benign bone tumor cases, of 137/211 (65\%) malignant bone tumors, of $73 / 353(20 \%)$ benign soft tissue tumors, of 56/219 (26\%) malignant soft tissue tumors, and of 72/356 (20\%) metastatic cases. The detailed analyses of the cases re-evaluated by BSTTC are shown in Fig. 1.

The diagnosis of some patients was changed after re-evaluation or histopathological examination. The findings of these patients are shown grouped as the initial diagnosis and definitive diagnosis in Fig. 2.

\section{DISCUSSION}

In comparison with data in the literature, some of the most frequently seen bone and soft tissue tumors seem to be quantitatively low in our series. The reason for this is that the multidisciplinary BSTTC deals more with the evaluation of diagnoses with a treatment process open to discussion. One of the frequently seen bone tumors in literature is enchondroma [2-4], which was seen in the current series as $8.6 \%$ of all bone tumors, and osteosarcoma was determined at the rate of $10.94 \%$.

The higher number of Ewing sarcoma than the relatively more often seen osteochondroma can be attributed to the tendency for the Council to evaluate malignant cases and there is a lesser need for the
Council in the diagnosis and treatment of benign masses. This was not very different for the soft tissue tumors in this study. For example, while cystic hygroma is among the most frequently seen soft tissue tumors [5], only 17 patients diagnosed with cystic hygroma were found in the 14-year archive of the BSTTC. The number of cases diagnosed with the malignant soft tissue tumor such as liposarcoma was 33, usually expected less common than cystic hygroma.

One of the aims of the multidisciplinary tumor council is to increase patient comfort and extend survival of these patients with collaboration between disciplines by organizing the optimum conditions for the treatment of patients with malignant bone or soft tissue tumors. In light of this information, the study data were examined of 1960 patients of 2397 who were first discussed by the Council and diagnosis was made for these 1960 patients. A total of 437 patients $(18.23 \%)$ were re-evaluated by the Council. The treatment protocols and survival rates of patients evaluated more than once by the Council, and the prognostic effect of the Council are targets for future studies.

When literature is examined, it can be seen that the treatment algorithm has generally been formed with a multidisciplinary approach for metastatic bone disease $[6,7]$. There are also studies related to a multidisciplinary approach for malignant bone tumors such as osteosarcoma and Ewing's sarcoma $[8,9]$. In a study that evaluated the multidisciplinary approach 
to 8 cases of malignant mass within the pelvis, satisfactory short-term results were obtained in all eight patients who received multidisciplinary treatment. According to that study, the survival rate of patients undergoing appropriate surgery for bone tumors in the pelvic region can be increased with a multidisciplinary approach comprising interventional radiologists, pediatric and medical oncologists, orthopedic surgeons, urologists, colorectal surgeons and plastic surgeons [10].

Re-opening the discussion of a case evaluated by the BSSTC is the result of a multidisciplinary approach. Since bone and soft tissue sarcomas are not common, there can be difficulties in the interpretation of imaging and histology, and because of the options of treatment methods and the complexity of treatment, systematic multidisciplinary team management is needed for these patients. A team with an integrated multidisciplinary clinic and a structured sarcoma tumor management panel facilitate team coordination and communication [11].

The management of soft tissue sarcomas requires multidisciplinary care [12]. When a multidisciplinary team is not used for patients with soft tissue sarcoma, surgery may not be optimal in the first intervention, and this can lead to a need for more extensive surgery than for the original tumor and radiation at later stages $[13,14]$. Also, there may be a more significant treatment costs for patients with bone and soft tissue tumors in the future because of the nonoptimal treatment process. Alamanda et al. [15] stated that there was an additional cost of 3679 USD in patients applied with re-excision following primary excision. Furthermore, primary operations performed without care by inexperienced hands can cause unnecessary amputations [16]. In the data of the current study, the three diagnoses most often repeatedly discussed were the metastatic bone disease, Ewing sarcoma, and osteosarcoma. When literature is examined, the importance of a multidisciplinary approach in the diagnosis and treatment of these types of malignant tumors can be observed [6-12].

It was observed that the diagnosis of some patients was changed after discussion in the Council or after histopathological examination. The findings of these patients were then shown as different patient groups with the initial and definitive diagnoses. In this section, when the tumors were separated into four groups according to the histopathological behavior, the most dramatic difference was in 27 patients with a benign diagnosis that became malignant. This can be considered to demonstrate that malignant tumors were captured at a lower grade and that the survival rate was increased with appropriate treatment. The diagnosis of malignant tumor of these 27 patients was confirmed with biopsy and surgery, and the importance of a multidisciplinary approach was observed on this point.

For the other groups, a less radical change was seen (initial benign diagnosis was definitively diagnosed as the benign and initial malignant diagnosis was definitively diagnosed as malignant). While 42 patients were considered to have an initial malignant diagnosis, a definitive benign diagnosis prevented unnecessary surgical interventions which would cause unnecessary costs, and this is another benefit of the multidisciplinary Council. In other words, in 69 (33.99\%) of 203 patients with a different definitive diagnosis from the initial diagnosis (a benign diagnosis becoming malignant and vice versa), the treatment was radically changed. As the ratio of all the patients evaluated by BSTTC, this number $(69 / 1960)$ constituted $3.52 \%$. This group can be considered to represent the patient group for whom the multidisciplinary tumor approach provided the most benefit.

When the diagnoses of the patients with the different initial diagnosis were examined, the three leading diagnoses that changed as a result of the detailed evaluation were formed of changes associated with infection, metastasis, and trauma. Several bone tumors are seen in forms similar to those of inflammation processes. The difference between osteomyelitis and primary bone tumor, especially Ewing sarcoma, always creates a problem. Consequently, it is generally difficult to interpret the difference between clinical and radiological findings. Osteomyelitis and bone tumors usually occur in young people. In several conditions, direct radiographs cannot be fully interpreted, and therefore, this method must not be significantly trusted when establishing the diagnosis. If a diagnosis is made by only direct radiographs, errors can be made [17].

\section{CONCLUSION}

In the treatment of a malignant tumor, working 
collaboration must be provided between the disciplines, and there must be specialists from radiology, pathology, medical oncology, radiation oncology and nuclear medicine in the team in addition to the orthopedic oncologist. In some patients thought to have a benign mass in the initial diagnosis, a diagnosis of a malignant mass was revealed as a result of the evaluation by the Tumour Council. Thus significant treatment changes were made in the early stages to prolong the survival rate of these patients, and the biopsychosocial healing process of the patients was accelerated. In some patients initially thought to have a malignant mass, a definitive diagnosis of a benign mass was revealed as a result of the Tumour Council evaluation, and for these patients unnecessary surgery, treatments and its costs were avoided.

\section{Authors' Contribution}

Study Conception: HSC, NB; Study Design: NB; Supervision: NB; Funding: HSC, NB; Materials: HG, FS; Data Collection and/or Processing: HSC, NB, HG, FS; Statistical Analysis and/or Data Interpretation: HG, FS; Literature Review: HSC; Manuscript Preparation: HSC and Critical Review: NB.

\section{Conflict of interest}

The authors disclosed no conflict of interest during the preparation or publication of this manuscript.

\section{Financing}

The authors disclosed that they did not receive any grant during conduction or writing of this study.

\section{Acknowledgments}

Authors thank to Funda COŞKUN for language editing.

\section{REFERENCES}

1. Yuceturk G, Sabah D, Kececi B, Kara AD, Yalcinkaya S. Prevalence of bone and soft tissue tumors. Acta Orthop Traumatol Turc 2011;45:135-43.

2. Hung Y, Ko W, Liu W, Chow C, Kwok Y, Wong C, et al. Local review of treatment of hand enchondroma (artificial bone substitute versus autologous bone graft) in a tertiary referral centre: 13 years' experience. Hong Kong Med J 2015;21:217-23.

3. Jewusiak EM, Spence KF, Sell KW. Solitary benign enchondroma of the long bones of the hand: Results of curettage and packing with freeze-dried cancellous-bone allograft. JBJS 1971;53:1587-90.

4. Picci P, Manfrini M, Fabbri N, Gambarotti M, Vanel D. Atlas of musculoskeletal tumors and tumorlike lesions: the Rizzoli case archive: Springer Science \& Business Media; 2014.

5. Hochman MG, Wu JS. MR imaging of common soft tissue masses in the foot and ankle. Magn Reson Imaging Clin N Am 2017;25:159-81.

6. Bechsgaard T, Lelkaitis G, Jensen KE, Ewertsen C. Diagnosis of bone metastasis from thyroid carcinoma: a multidisciplinary approach. Acta Radiol Open 2015;4:2058460115603248.

7. Satoh T, Ishiyama H. [Current diagnosis and the multidisciplinary approach to the treatment of bone metastases in patients with prostate cancer]. Nihon Rinsho. 2016;74(1):129-36. [Aeticle in Japanese]

8. Gaspar N, Hawkins DS, Dirksen U, Lewis IJ, Ferrari S, Le Deley M-C, et al. Ewing sarcoma: current management and future approaches through collaboration. J Clin Oncol 2015;33:3036-46.

9. Wittig JC, Bickels J, Priebat D, Jelinek J, Kellar-Graney K, Shmookler B, et al. Osteosarcoma: a multidisciplinary approach to diagnosis and treatment. Am Fam Physician 2002;65:1123-32. 10. Yokoyama R, Beppu Y, Tobisu K-i, Moriya Y, Uchiyama K, Kito $\mathrm{M}$, et al. A multidisciplinary approach to the treatment of malignant pelvic bone tumors: results with eight consecutive patients. J Orthop Sci 2000;5:449-56.

11. Siegel GW, Biermann JS, Chugh R, Jacobson JA, Lucas D, Feng M, et al. The multidisciplinary management of bone and soft tissue sarcoma: an essential organizational framework. J Multidiscip Healthc 2015;8:109-15.

12. Papagelopoulos PJ, Mavrogenis AF, Mastorakos DP, Patapis P, Soucacos PN. Current concepts for management of soft tissue sarcomas of the extremities. J Surg Orthop Adv 2008;17:204-15. 13. Siebenrock KA, Hertel R, Ganz R. Unexpected resection of soft-tissue sarcoma. More mutilating surgery, higher local recurrence rates, and obscure prognosis as consequences of improper surgery. Arch Orthop Trauma Surg 2000;120:65-9.

14. Umer HM, Umer M, Qadir I, Abbasi N, Masood N. Impact of unplanned excision on prognosis of patients with extremity soft tissue sarcoma. Sarcoma 2013;2013:498604.

15. Alamanda VK, Delisca GO, Mathis SL, Archer KR, Ehrenfeld JM, Miller MW, et al. The financial burden of reexcising incompletely excised soft tissue sarcomas: a cost analysis. Ann Surg Oncol 2013;20:2808-14

16. Venkatesan M, Richards C, McCulloch T, Perks A, Raurell A, Ashford R, et al. Inadvertent surgical resection of soft tissue sarcomas. Eur J Surg Oncol 2012;38:346-51.

17. Coventry MB. The differential diagnosis of malignant bone tumors. Ann Surg 1950;132:888-98. 